\title{
The Advancing Training in Russian for the Special Purposes of Foreign Students of a Pre-University Level
}

\author{
Alla Kulik ${ }^{1, a *}$ \\ 1 Peoples' Friendship University of Russia, Faculty of Russian Language and General \\ Education Disciplines, 117198, 6 Miklukho-Maclay str., Moscow, Russia \\ apesochek08@mail.ru \\ ${ }^{*}$ Corresponding author
}

Keywords: advancing training, professional module, humanitarian profile, content of education, a pre-university level

\begin{abstract}
The paper speaks in favor of the advancing introduction of the professional module to the practice of teaching Russian by foreign pupil of a humanitarian profile at the elementary level (A1). It is established that the professionalization of educational process within a practical course of Russian needs to begin from first months of training. The created linguodidactic system is directed on improving the professionally focused content of education, on ensuring the quality of training of foreign students (who are not philologists).
\end{abstract}

\section{Introduction}

In the Russian national doctrine of education, which found reflection in the Federal Law on Education in the Russian Federation (2012) and in the new "Requirements for the development of the additional general education programs providing training of foreign citizens and stateless persons for development of professional general education programs in Russian" (2014), the prime attention is paid to a problem of quality of training of future expert which predicted portrait is characterized multilateral education, creative thinking, high level professional, intercultural communicative and speech competences, ability to join in the international scientific and professional and cultural context.

Integration of Russia into the world community stimulates also the process of modernization of the Russian educational system. As one of necessary conditions of such integration compliance of training of foreign students on Russian to the international standards of professionalism which integral part is communicative competence acts.

Today, the innovative education at the higher school directed on the advancing training in subjects including to Russian as foreign (it is recorded in the documents accepted by UNESCO), on formation at future experts not only a certain knowledge and abilities, but also the competences focused on the ability to apply them in practical activities develops.

It should be noted that L.S. Vygotsky's doctrine about the two levels of intellectual development is the theoretical base on which the idea of the advancing training is based. According to L.S. Vygotsky's doctrine, training, i.e. leaning on the reached level of development, has to be ahead of a student, stimulate, and lead the process of mastering knowledge needs to be organized so that to bring new elements and form new relations, ensuring development.

For the first time, we prove the advancing introduction of the professional module to practice of teaching Russian by the foreign pupil of a humanitarian profile already at the elementary level (A1). We believe that the professionalization of educational process within a practical course of Russian needs to begin from the very first months of training. It provides a chance to seize the content of micro and macro modules, which are switched on in the professional module.

\section{Research relevance}

Relevance of the chosen subject and our research is caused by: 
1) The need of not language higher education institutions for modern technologies of training in Russian as foreign, the modern state standards corresponding to purposes;

2) An insufficient readiness of the linguistical and methodical concept of professionally focused on foreign students' training in Russian at the initial stage;

3) An innovative content of students education in humanities taking into account the professional module (A1, A2 levels) in a practical course of Russian.

\section{Key issues in advancing training}

Acquaintance to the materials happens already in the first semester. Traditionally, the beginning of classes depends on terms of input of general education subjects, on the directions. We suggest to begin a propaedeutic course with the fourth week of training, when the elementary level of proficiency of speech communication in Russian is already achieved. Further, it should go for six hours since the ninth week and until the end of the first semester. In the second semester, the number of hours increases to eight (twelve) per one week.

An analysis of subjects and situations of training the scientific speech style using special language materials allowed to identify a total of two groups of functions. The first one is connected with the organization of pedagogical process. The second one is connected with the implementation of the speech statement.

The first group of functions covers topics and situations that act as a unit of organization of language and speech material, means and method of organizing utterances, means and methods of teaching, exercise, stimulus for speech, methodical conditions, without which students cannot learn and manage communication activities, methods of systematization of language and speech material, means and method of control, which serve as a factor that determines the educational and educational orientation of the training content.

The second group of functions takes place when the topic and the situation are implemented as a starting point in the deployment of the content of speech, indicating the boundary of the statement, the method of differentiating the distinction between phenomena of reality, the subject of speech, a guide to the approximate content of speech.

In the content of training, the topic determines the composition of the vocabulary serving the topic.

The subject of speech activity is the topic, which involves a set of topics within which students should communicate, and, according to the principle of minimization, should be limited to the content. Such a restriction regulates both the composition and volume of the linguistic, primarily lexical, material, as well as the meaningful aspect of speech, thereby ensuring the practical, educating, and developing aspects of learning.

The topic acts as a means and method of systematizing speech and language materials, as a means of systematizing objects of speech in the course of Russian as a foreign language, which is reflected in the program and in textbooks. For the purposes of systematization, the topic is implemented in different hierarchical units: in a generalized unit - in the program, in a fractional unit - in the textbook. As a means of lexical material, the topic is implemented in various kinds of exercises, for example, in exercises on the compilation of subject groups.

In training, a topic acts not as a goal, but as a means of teaching speech activity. The situation is both a learning tool and its purpose [1].

We offer the advanced presentation of educational texts with a professional theme, i.e. prior to studying them in the subject course, and we recommend starting the propaedeutic introductory-subject course, as already noted above, from the fourth week of study [2].

A.N. Baranov revealed the frequency hierarchy of the five most important topics in the texts on Politic: (1) the functioning of the legislature (2,430 publications); (2) the functioning of the executive branch $(1,834$ 
publications); (3) regional policy (1,317 publications); (4) crime (1,189 publications); (5) reforming natural monopolies (965 publications) [3].

A newspaper text is an interpretation of fragments of public life: facts, events, phenomena, personalities; therefore, it may be viewed as a motivated and targeted axiological version of fragments of social life [4].

Within the theme, it is possible to group real texts intended for teaching reading and listening, and texts that should be generated in the process of oral or written utterance in accordance with the substantive content of these texts, that is, the topic allows to correlate these texts with non-linguistic reality and our knowledge of her [5-9].

Thus, the text (considered by us both as a component and as the material basis of the content of vocationally oriented education) serves as another component of the content of learning Russian as a foreign language. Educational (stable) texts provide, as a rule, basic knowledge of the topic. Depending on the nature of the topic, that is, the ratios of stable and variable components in it, learning (stable) texts can be represented by several types. These may be texts: (i) giving a theoretical coverage of the topic; (ii) containing historical background on the topic; (iii) describing a particular historical fact or event, particularly significant in the framework of this topic; (iv) being reference-encyclopedic in nature.

Typical texts may contain a description, message, evidence, reasoning. Based on the fact that scientific texts serve the sphere of human cognitive activity, it appears that it is the language of science that can serve as the basis for teaching the speech culture of humanitarian students. A scientific text can serve as the basis for the formation of the speech culture of modern specialists in the Humanities. However, along with the identification of common patterns that determine the communicative nature of the text in general, it is important for humanitarian students to form an idea of the specifics of a scientific text as a special form of communication.

Students should know the basic rules for constructing definitions. A typical structure of a definition includes the basic identification signs of a concept, phenomenon or object, and their location is determined by the inductive principle: the definition begins with generic signs introducing the defined concept, phenomenon, or object into a circle of homogeneos objects, and ends with a characteristic or property inherent exclusively to this definition. The ability to see the structure of the definition, to identify the identification features of this concept, to realize the hierarchy of constructing the latter develops a number of skills for students, such as (i) the correct use of terminology and (ii) the need for accurate and coherent presentation of scientific knowledge. The universal features of texts in general and, of scientific texts in particular are informative, modality, coherence, contraction, consistency, and articulation [10-12].

What are the same themes in different sciences or within the same science? In such branches of knowledge topics may coincide in terms of objects. It is known that objects in a number of sciences are common, but in each of these sciences they are considered from different points of view. On the other hand, different subjects of study in individual sciences can be viewed in the same planes.

It may be noted that such areas of research that are common to applied sciences include: (i) a general concept of the subject of this science (in its specific varieties); (ii) species varieties of objects; (iii) the most important characteristics of objects; (iv) object structure; (v) connections, relationships, dependencies between objects, their parts, their properties; (vi) measurement of objects: (a) units of measurement, (b) methods of measurement; (vii) use of objects: (a) purpose, (b) scope; (viii) obtaining objects for use: (a) sources of receipt; (b) methods of production, (c) a practical process of obtaining [13].

\section{Conclusion}

As is known, an educational text is a unit of text-based learning, text-based educational communication [14]. We believe that the training text is a special type of secondary text, the specificity of which is determined by its features, which consist in the fact that it is limited to the selected and minimized 
language in accordance with the target setting. For a teacher, a text is a learning tool, and for a student, an object. Consequently, the peculiarity of the text in terms of its attitude to the educational process is that it is both an object of study and a means of learning.

Thus, in this part of the study has identified the following: (i) themes and texts that allow for advanced professional-oriented training of foreign non-philology students; (ii) subjects and texts are micromodules included in the structure of education; (iii) originality of the text in terms of its attitude to the educational process. Also, the paper clearly shows that the text is characterized as (i) a component and a material basis of the content of vocationally oriented training; (ii) an object of study and a means of teaching speech activity and the formation of reading mechanisms; (iii) considered typical texts: description, communication, proof, reasoning, and the structure of the definition.

In conclusion, updating and increasing speech materials with the early introduction of the scientificstyle speech on the material of the specialty language in the initial stage of training requires the organization of innovative, integrated, modular training courses based on well-developed educational material that provides for using both traditional and professionally oriented technologies [15].

\section{References}

[1] Klimentenko, A. D. (1984). The content of training in foreign languages at high school (pp. 127-133). Moscow, Russia: Pedagogic.

[2] Kulik, A. D. (2008). Innovation in creation of an educational and methodical complex on scientific style of the speech at the initial stage of training of foreign students in system of high school education. In Optimization of process of training of foreign citizens in the conditions of modernization of the higher education in Russia: materials of the international scientific and practical conference (September 25-27, 2008) (pp. 37-44). Lipetsk, Russia.

[3] Baranov, A. N. (2008). About one complex method of an analysis of a political discourse. In Language of mass media as an object of interdisciplinary research: materials of the 2nd international conference (February 14-16, 2008). Moscow, Russia: MAX. Press.

[4] Kirilenko, M. A. (2008). Stylistic features of newspaper texts during parliamentary election campaign. In Language of mass media as an object of interdisciplinary research: materials of the 2nd international conference (February 14-16, 2008). Moscow, Russia: MAX. Press.

[5] Bim, I. L. (1977). Methodics of training in a foreign language as science and problems of the school textbook. Moscow, Russia: Russian language.

[6] Vyatyutnev, M. N. (1984). Theory of the textbook of Russian language as foreign (methodical bases). Moscow, Russia: Russian language.

[7] Mirolyubov, A. A., \& Iyevleva, Z. N. (1976). The initial stage in training in nonnative language and a way of its allocation. A. A. Mirolyubov, E. A. Sosenko (Ed.), Questions of training in Russian of foreigners at the initial stage (pp. 205-212). Moscow, Russia.

[8] Passov, E. I. (1989). Bases of a communicative method of training in foreign-language communication. Moscow, Russia.

[9] Shatilov, S. F. (1976). On the discussion about speech and language skills. Foreign Languages at the Higher School, 2, pp. 12-17.

[10] Gzlperin, I. R. (1981). Text as object of linguistic research. Moscow, Russia: Nauka.

[11] Zarubina, N. D. (1981). Linguistic and historical aspects. Moscow, Russia: Russian language.

[12] Mets, N. A., Mitrofanova, O. D., \& Odintsova, O. D. (1981). Structure of the scientific text and training of the monological speech. Moscow, Russia.

[13] Motina, E. I. (1983). Language and specialty: linguistical methodical bases of training in Russian of 
the students of non-philological profiles (pp. 51-52). Moscow, Russia: Russian language.

[14] Babaylova, A. E. (1987). The text as a product, means and object of communication when training in nonnative language. Saratov, Russia.

[15] Kulik, A. D. (2016). Integrative and modular approach to professionally focused training in Russian foreign students (initial stage). Moscow, Russia. 